PROCEEDINGS OF THE

AMERICAN MATHEMATICAL SOCIETY

Volume 134, Number 3, Pages 637-644

S 0002-9939(05)08128-1

Article electronically published on August 29, 2005

\title{
THE AMENABILITY AND NON-AMENABILITY OF SKEW FIELDS
}

\author{
GÁBOR ELEK \\ (Communicated by Martin Lorenz)
}

\begin{abstract}
We investigate the amenability of skew field extensions of the complex numbers. We prove that all skew fields of finite Gelfand-Kirillov transcendence degree are amenable. However there are both amenable and non-amenable finitely generated skew fields of infinite Gelfand-Kirillov transcendence degree.
\end{abstract}

\section{INTRODUCTION}

Definition 1.1. Let $k$ be a commutative field and let $\mathcal{A}$ be a unital $k$-algebra. We say that $\mathcal{A}$ is amenable if for any finite subset $\left\{r_{1}, r_{2}, \ldots, r_{n}\right\} \subset \mathcal{A}$ and real number $\epsilon>0$ there exists a finite-dimensional $k$-subspace $V \subset \mathcal{A}$ such that

$$
\frac{\operatorname{dim}_{k}\left(\sum_{i=1}^{n} r_{i} V\right)}{\operatorname{dim}_{k} V}<1+\epsilon .
$$

In [5], we saw that the group algebra of an amenable group is amenable, and the group algebra of the free group of two generators is non-amenable. Any affine algebra of subexponential growth or any commutative algebra is amenable as well. It is easy to see that amenable algebras satisfy the invariant basis number property. In this paper, we investigate the amenability of skew field extensions. Our main result is the following theorem.

\section{Theorem 1.}

(a) If $k \subseteq D$ is a skew field that is finite dimensional over its center, then it is amenable.

(b) If $k \subseteq D$ has finite Gelfand-Kirillov transcendence degree, then it is amenable.

(c) There exist finitely generated amenable skew fields of infinite GelfandKirillov transcendence degree.

(d) If $k \subseteq D \subseteq E$ are skew fields and $D$ is non-amenable, then $E$ is nonamenable as well.

(e) The free field of Cohn [4 is non-amenable.

Received by the editors November 24, 2003 and, in revised form, June 15, 2004 and October 4, 2004 .

2000 Mathematics Subject Classification. Primary 12E15, 43A07.

Key words and phrases. Skew fields, amenable algebras, Gelfand-Kirillov transcendence degree, von Neumann algebras. 
In the course of the proof we shall see that $\mathbb{C}(\Gamma)$ is an amenable $\mathbb{C}$-algebra if and only if $\Gamma$ is an amenable group. The corresponding conjecture for general fields remains open.

\section{The Ore-Property AND Amenability}

Lemma 2.1. If $\mathcal{A}$ is an amenable domain, then it has the left Ore-property that is for any non-zero $a, b \in \mathcal{A} ; a \mathcal{A} \cap b \mathcal{A} \neq 0$.

Proof. There exists a finite dimensional subspace $W \subseteq \mathcal{A}$ such that $\operatorname{dim}_{k}(a W \cap W)$ $>\frac{1}{2} \operatorname{dim}_{k}(W)$ and $\operatorname{dim}_{k}(b W \cap W)>\frac{1}{2} \operatorname{dim}_{k}(W)$, hence $a W \cap b W \neq 0$.

Consequently, if $\mathcal{A}$ is an amenable domain, then one can consider its classical ring of quotient $\widetilde{\mathcal{A}}$, a skew field.

Proposition 2.2. If $\mathcal{A}$ is an amenable domain, then $\widetilde{\mathcal{A}}$ is amenable as well.

Proof. Let $x_{1}, x_{2}, \ldots, x_{m} \in \widetilde{\mathcal{A}}$. Then by the definition of the classical ring of quotient there exists a non-zero element $r \in \mathcal{A}$ such that $x_{1} r, x_{2} r, \ldots, x_{m} r \in \mathcal{A}$. By the amenability of $\mathcal{A}$ there exists a sequence of finite-dimensional $k$-vector spaces $W_{n} \subseteq \mathcal{A}$, such that

$$
\lim _{n \rightarrow \infty} \frac{\operatorname{dim}_{k}\left(\sum_{i=1}^{m} x_{i} r W_{n}\right)}{\operatorname{dim}_{k}\left(W_{n}\right)}=1 .
$$

Lemma 2.3. Let $Z_{n}=\left\{v \in W_{n}: x_{i} r v \in W_{n}\right.$ for all $\left.i\right\}$. Then $\lim _{n \rightarrow \infty} \frac{\operatorname{dim}_{k}\left(Z_{n}\right)}{\operatorname{dim}_{k}\left(W_{n}\right)}=$ 1.

Proof. By (2), for any $1 \leq i \leq m, \lim _{n \rightarrow \infty} \frac{\operatorname{dim}_{k}\left(x_{i} r W_{n} \cap W_{n}\right)}{\operatorname{dim}_{k}\left(W_{n}\right)}=1$. Hence if $Z_{n}^{i}=$ $\left\{v \in W_{n}, x_{i} r v \in W_{n}\right\}$, then $\lim _{n \rightarrow \infty} \frac{\operatorname{dim}_{k}\left(Z_{n}^{i}\right)}{\operatorname{dim}_{k}\left(W_{n}\right)}=1$. Since $Z_{n}=\bigcap_{i=1}^{m} Z_{n}^{i}$, the lemma follows.

Obviously, if $T_{n}=\left\{v \in W_{n}: r v \in W_{n}\right\}$, then $\lim _{n \rightarrow \infty} \frac{\operatorname{dim}_{k}\left(T_{n}\right)}{\operatorname{dim}_{k}\left(W_{n}\right)}=1$. Thus if $S_{n}=T_{n} \cap Z_{n}$, then $\lim _{n \rightarrow \infty} \frac{\operatorname{dim}_{k}\left(S_{n}\right)}{\operatorname{dim}_{k}\left(W_{n}\right)}=1$, and $\lim _{n \rightarrow \infty} \frac{\operatorname{dim}_{k}\left(r S_{n}\right)}{\operatorname{dim}_{k}\left(W_{n}\right)}=1$. Now if $v \in r S_{n}$, then $v \in W_{n}$ and for any $1 \leq i \leq m, x_{i} v \in W_{n}$, that is, $r S_{n} \subseteq W_{n}$ and $x_{i} r S_{n} \subseteq W_{n}$. Since $\lim _{n \rightarrow \infty} \frac{\operatorname{dim}_{k}\left(W_{n}\right)-\operatorname{dim}_{k}\left(r S_{n}\right)}{\operatorname{dim}_{k}\left(W_{n}\right)}=0$, it follows that

$$
\lim _{n \rightarrow \infty} \frac{\operatorname{dim}_{k}\left(\sum_{i=1}^{m} x_{i} W_{n}\right)}{\operatorname{dim}_{k}\left(W_{n}\right)}=1,
$$

proving the amenability of $\widetilde{\mathcal{A}}$.

Note that by our previous proposition the classical ring of fraction of the Weylalgebras are amenable. Let us recall the notion of the Gelfand-Kirillov transcendence degree [6].

Definition 2.4. Let $D$ be a skew field extension of the commutative field $k$. Then

$$
G K-\operatorname{tr} \operatorname{deg}(D)=\sup _{V} \inf _{0 \neq r} \limsup _{n \rightarrow \infty} \frac{\log \operatorname{dim}_{k}\left((k+V r)^{n}\right)}{\log n},
$$

where $V$ runs through all the finite dimensional subspaces of $D$ containing the unit.

Note that GK-tr $\operatorname{deg}(D)<\infty$ if and only if there exists a $d>0$ with the following property: For any $c_{1}, c_{2}, \ldots, c_{m} \in D$ there exists $r \in D$ such that the affine algebra generated by $1, c_{1} r, c_{2} r, \ldots, c_{m} r$ has polynomial growth with GelfandKirillov dimension not greater than $d$. 
Proposition 2.5. If $D$ has finite Gelfand-Kirillov transcendence degree, then $D$ is amenable.

Proof. We prove that if for any subset $c_{1}, c_{2}, \ldots, c_{m} \in D$ there exists $r \in D$ such that $1, c_{1} r, c_{2} r, \ldots, c_{m} r$ generate an amenable domain, then $D$ is amenable. Indeed, if

$$
\lim _{n \rightarrow \infty} \frac{\operatorname{dim}_{k}\left(\sum_{i=1}^{m} c_{i} r W_{n}\right)}{\operatorname{dim}_{k}\left(W_{n}\right)}=1,
$$

then

$$
\lim _{n \rightarrow \infty} \frac{\operatorname{dim}_{k}\left(\sum_{i=1}^{m} c_{i} V_{n}\right)}{\operatorname{dim}_{k}\left(V_{n}\right)}=1
$$

where $V_{n}=r W_{n}$. Thus $D$ is in fact amenable.

Note however, that Aizenbud proved in 1 that for some poly-cyclic groups $\Gamma$ the classical ring of quotient of the group algebra $k(\Gamma)$ has infinite Gelfand-Kirillov transcendence degree. Since such groups are amenable, these finitely generated skew fields above are amenable as well.

Also, since all affine commutative algebras and all matrix algebras over such algebras are of polynomial growth, we have the following corollary:

Proposition 2.6. If $k \subseteq L$ is finite dimensional over its center, then $L$ is amenable.

The class of amenable skew fields are closed under some operations:

\section{Proposition 2.7.}

(a) The direct limit of amenable skew fields is amenable.

(b) If $k \subseteq D$ and any finitely generated subskewfield $E \subseteq D$ is amenable, then $D$ is amenable as well.

(c) If $A$ and $B$ are amenable skew fields and $A \otimes_{k} B$ is a domain, then $A \otimes_{k} B$ is amenable, hence by Proposition $2.2 \widetilde{A \otimes_{k} B}$ is also an amenable skew field.

Proof. Parts (a) and (b) are easy exercises; for (c), let us consider the amenable $k$ algebras $A$ and $B$. It is enough to prove that if $a_{1}, a_{2}, \ldots, a_{l} \in \mathcal{A}, b_{1}, b_{2}, \ldots, b_{m} \in B$, then there exists a sequence of finite-dimensional subspaces $\left\{V_{n}\right\} \subseteq A \otimes_{k} B$ such that

$$
\lim _{n \rightarrow \infty} \frac{\operatorname{dim}_{k}\left(\sum_{i=1}^{l} \sum_{j=1}^{m}\left(a_{i} \otimes b_{j}\right) V_{n}\right)}{\operatorname{dim}_{k}\left(V_{n}\right)}=1 .
$$

Let $\left\{W_{n}\right\} \subseteq A,\left\{Z_{n}\right\} \subseteq B$ be sequences of finite-dimensional subspaces such that

$$
\lim _{n \rightarrow \infty} \frac{\operatorname{dim}_{k}\left(\sum_{i=1}^{l} a_{i} W_{n}\right)}{\operatorname{dim}_{k}\left(W_{n}\right)}=1
$$

and

$$
\lim _{n \rightarrow \infty} \frac{\operatorname{dim}_{k}\left(\sum_{j=1}^{m} b_{j} Z_{n}\right)}{\operatorname{dim}_{k}\left(Z_{n}\right)}=1 .
$$

Set $V_{n}=W_{n} \otimes Z_{n}$. Then

$$
\left(\sum_{i=1}^{l} \sum_{j=1}^{m}\left(a_{i} \otimes b_{j}\right) V_{n}\right) \subseteq\left(\sum_{i=1}^{l} a_{i} W_{n}\right) \otimes\left(\sum_{j=1}^{m} b_{j} Z_{n}\right),
$$

hence the proposition follows. 


\section{NON-AMENABLE SUBSKEWFIELDS}

Proposition 3.1. Let $k \subseteq D \subseteq E$ be skewfields. Suppose that $D$ is non-amenable; then $E$ is non-amenable as well.

Proof. If $D$ is non-amenable, then there exists $\alpha>1$ and $g_{1}, g_{2}, \ldots, g_{l} \in D$ such that for any finite-dimensional $k$-subspace $W \subseteq D$

$$
\operatorname{dim}_{k}\left(\sum_{i=1}^{l} g_{i} W\right) \geq \alpha \operatorname{dim}_{k}(W) .
$$

It is enough to prove that for any $m \geq 1$ and any finite-dimensional $k$-subspace $Z$ of the $m$-dimensional left $D$-module $\bigoplus_{j=1}^{m} D$,

$$
\operatorname{dim}_{k}\left(\sum_{i=1}^{l} g_{i} Z\right) \geq \alpha \operatorname{dim}_{k}(Z) .
$$

We proceed by induction. The $m=1$ case is just (3) . Suppose that (4) holds for $m=1,2, \ldots, n$. Let $Z \subseteq \bigoplus_{j=1}^{n+1} D$ and let $\pi^{n+1}: \bigoplus_{j=1}^{n+1} D \rightarrow D$ be the projection onto the last coordinate. If $\operatorname{dim}_{k}\left(\pi^{n+1}(Z)\right)=\operatorname{dim}_{k}(Z)$, then

$$
\operatorname{dim}_{k}\left(\sum_{j=1}^{l} g_{j} Z\right)=\operatorname{dim}_{k}\left(\sum_{j=1}^{l} g_{j}\left(\pi^{n+1}(Z)\right)\right) \geq \alpha \operatorname{dim}_{k}(Z) .
$$

If $\operatorname{dim}_{k}\left(\pi^{n+1}(Z)\right)<\operatorname{dim}_{k}(Z)$, then $Z=Z_{1} \oplus Z_{2}$ as $k$-subspaces, where $Z_{1} \subseteq$ $\bigoplus_{j=1}^{n} D$ and $\operatorname{dim}_{k}\left(\pi^{n+1}(Z)\right)=\operatorname{dim}_{k}\left(Z_{2}\right)$. Let $v_{1}, v_{2}, \ldots, v_{s_{1}}$ be a $k$-basis for $Z_{1}$ and let $w_{1}, w_{2}, \ldots, w_{s_{2}}$ be a $k$-basis for $Z_{2}$. Let $G$ be the $k$-vector space generated by $\left\{g_{1}, g_{2}, \ldots, g_{l}\right\}$. Then by the inductional hypothesis, there exist elements

$$
\begin{aligned}
& g_{j}^{i} \in G, 1 \leq i \leq t_{1}, 1 \leq j \leq s_{1}, \\
& h_{j}^{i} \in G, 1 \leq i \leq t_{2}, 1 \leq j \leq s_{2},
\end{aligned}
$$

such that $t_{1} \geq \alpha s_{1}, t_{2} \geq \alpha s_{2}$, and

$$
\left\{z_{i}=\sum_{j=1}^{s_{1}} g_{j}^{i} v_{j}\right\}_{i=1,2, \ldots, t_{1}}
$$

and

$$
\left\{z_{i}^{\prime}=\sum_{j=1}^{s_{2}} h_{j}^{i} \pi^{n+1}\left(w_{j}\right)\right\}_{i=1,2, \ldots, t_{2}}
$$

are both independent systems of vectors in $\bigoplus_{i=1}^{n} D$, resp. $\pi^{n+1}\left(\bigoplus_{i=1}^{n+1} D\right)$. Then $\left\{z_{i}=\sum_{j=1}^{s_{1}} g_{j}^{i} v_{j}\right\} \cup\left\{z_{i}^{\prime \prime}=\sum_{j=1}^{s_{2}} h_{j}^{i}\left(w_{j}\right)\right\}$ form an independent system of more than $\alpha \cdot \operatorname{dim}_{k}(Z)$ elements, proving (4) for the subspace $Z \subseteq \bigoplus_{j=1}^{n+1} D$.

\section{The CONSTRUCTiOn of A NON-AMENABLE SKEW FIELD}

Definition 4.1. Let $\Gamma$ be a discrete group and let $V$ be a vector space over $k$. A representation $\phi: \Gamma \rightarrow G L(V)$ is called algebraically amenable, if for any 
$g_{1}, g_{2}, \ldots, g_{m} \in \Gamma$ and $\epsilon>0$ there exists a finite-dimensional vector space $W \subseteq V$ such that

$$
\frac{\operatorname{dim}_{k}\left(\sum_{i=1}^{m} g_{i} W\right)}{\operatorname{dim}_{k}(W)} \leq 1+\epsilon
$$

Note that if $\mathcal{A}$ is an amenable $k$-algebra, then the group of invertible elements of $\mathcal{A}$ acts on $\mathcal{A}$ in an algebraically amenable fashion. We should observe that the group of non-zero elements of an amenable skew field can in fact be a non-amenable group. As a matter of fact it is Lichtman's conjecture that any skew field which is infinite dimensional over its center contains a free group of two generators. By the result of Makar-Limanov [10] the first Weyl skew field even contains a free group algebra as a subalgebra.

Proposition 4.2. Let $\Gamma$ be a countable group represented by unitary transformations on a Hilbert-space $\mathcal{H}$. If the representation is algebraically amenable, then it is amenable in the sense of Bekka [2], that is, there exists a linear map $\tau: B(\mathcal{H}) \rightarrow \mathbb{C}$ from the space of bounded linear operators to the complex numbers such that $\tau(I d)=1$ and

$$
\tau(A)=\tau\left(g^{-1} A g\right)
$$

for any $A \in B(\mathcal{H}), g \in \Gamma$.

Proof. By our assumption, there exists a sequence of finite-dimensional subspaces $W_{n} \subseteq \mathcal{H}, n \geq 1$, such that for any $g \in \Gamma$,

$$
\lim _{n \rightarrow \infty} \frac{\operatorname{dim}_{\mathbb{C}}\left(g W_{n}+W_{n}\right)}{\operatorname{dim}_{\mathbb{C}}\left(W_{n}\right)}=1 .
$$

Let $P_{n}$ denote the orthogonal projection onto $W_{n}$. Then for any $A \in B(\mathcal{H})$,

$$
\frac{\operatorname{Tr}\left(P_{n} A P_{n}\right)}{\operatorname{dim}_{\mathbb{C}}\left(W_{n}\right)}
$$

is a sequence of complex numbers bounded by the norm of $A$. Hence one can consider

$$
\tau(A)=\lim _{\omega} \frac{\operatorname{Tr}\left(P_{n} A P_{n}\right)}{\operatorname{dim}_{\mathbb{C}}\left(W_{n}\right)}
$$

where $\omega$ is an ultrafilter on the natural numbers and $\lim _{\omega}$ is the corresponding ultralimit. Then the map $\tau: B(\mathcal{H}) \rightarrow \mathbb{C}$ is clearly bounded and linear. In order to prove that $\tau(A)=\tau\left(g A g^{-1}\right)$, it is enough to see that for any $g \in \Gamma$ and $A \in B(\mathcal{H})$,

$$
\lim _{n \rightarrow \infty} \frac{\operatorname{Tr}\left(P_{n} A P_{n}\right)-\operatorname{Tr}\left(P_{n} g^{-1} A g P_{n}\right)}{\operatorname{dim}_{\mathbb{C}}\left(W_{n}\right)}=0 .
$$

Let $G_{n}=g^{-1}\left(g W_{n} \cap W_{n}\right)$. Then $\lim _{n \rightarrow \infty} \frac{\operatorname{dim}_{\mathbb{C}}\left(G_{n}\right)}{\operatorname{dim}_{\mathbb{C}}\left(W_{n}\right)}=1$ and both $G_{n}$ and $g G_{n}$ are subspaces of $W_{n}$. Pick an orthonormal basis $\left\{v_{1}, v_{2}, \ldots, v_{k_{n}}\right\}$ for $G_{n}$ and extend it to an orthonormal basis for the whole $W_{n}$ by adding $w_{1}, w_{2}, \ldots, w_{l_{n}}$, where $k_{n}=\operatorname{dim}_{\mathbb{C}}\left(G_{n}\right), l_{n}=\operatorname{dim}_{\mathbb{C}}\left(W_{n}\right)-\operatorname{dim}_{\mathbb{C}}\left(G_{n}\right)$.

Let $g_{n}$ be a unitary transformation on $W_{n}$ such that $\left.g_{n}\right|_{G_{n}}=g$. Define $g_{n}$ to be the identity transformation on $W_{n}^{\perp}$. Then

$$
\operatorname{Tr}\left(P_{n} g_{n}^{-1} A g_{n} P_{n}\right)=\operatorname{Tr}\left(P_{n} A P_{n}\right) .
$$

Indeed, $g_{n}$ commutes with $P_{n}$, hence

$$
\operatorname{Tr}\left(P_{n} g_{n}^{-1} A g_{n} P_{n}\right)=\operatorname{Tr}\left(g_{n}^{-1} P_{n} A P_{n} g_{n}\right)
$$


and

$$
\operatorname{Tr}\left(g_{n}^{-1} P_{n} A P_{n} g_{n}\right)=\operatorname{Tr}\left(P_{n} A P_{n}\right)
$$

by the property of the trace.

Lemma 4.3.

$$
\lim _{n \rightarrow \infty} \frac{\operatorname{Tr}\left(P_{n} g_{n}^{-1} A g_{n} P_{n}\right)-\operatorname{Tr}\left(P_{n} g^{-1} A g P_{n}\right)}{\operatorname{dim}_{\mathbb{C}}\left(W_{n}\right)}=0 .
$$

Proof. Note that

$$
\begin{aligned}
\operatorname{Tr}\left(P_{n} g^{-1} A g P_{n}\right) & =\sum_{i=1}^{k_{n}}\left\langle g^{-1} A g\left(v_{i}\right), v_{i}\right\rangle+\sum_{i=1}^{l_{n}}\left\langle g^{-1} A g\left(w_{i}\right), w_{i}\right\rangle \\
& =\sum_{i=1}^{k_{n}}\left\langle A g\left(v_{i}\right), g\left(v_{i}\right)\right\rangle+\sum_{i=1}^{l_{n}}\left\langle A g\left(w_{i}\right), g\left(w_{i}\right)\right\rangle .
\end{aligned}
$$

Similarly,

$$
\operatorname{Tr}\left(P_{n} g_{n}^{-1} A g_{n} P_{n}\right)=\sum_{i=1}^{k_{n}}\left\langle A g_{n}\left(v_{i}\right), g_{n}\left(v_{i}\right)\right\rangle+\sum_{i=1}^{l_{n}}\left\langle A g_{n}\left(w_{i}\right), g_{n}\left(w_{i}\right)\right\rangle .
$$

Since $g_{n}\left(v_{i}\right)=g\left(v_{i}\right)$, we have the following estimate:

$$
\begin{aligned}
& \left|\operatorname{Tr}\left(P_{n} g_{n}^{-1} A g_{n} P_{n}\right)-\operatorname{Tr}\left(P_{n} g^{-1} A g P_{n}\right)\right| \\
& \quad \leq \sum_{i=1}^{l_{n}}\left(\left|\left\langle A g_{n}\left(w_{i}\right), g_{n}\left(w_{i}\right)\right\rangle\right|+\left|\left\langle A g\left(w_{i}\right), g\left(w_{i}\right)\right\rangle\right| \leq 2\|A\| \cdot l_{n} .\right.
\end{aligned}
$$

Therefore the lemma follows from the fact that

$$
\lim _{n \rightarrow \infty} \frac{l_{n}}{\operatorname{dim}_{\mathbb{C}}\left(W_{n}\right)}=0 .
$$

Consequently, by the Lemma 4.3 and (5) we can see that $\tau(A)=\tau\left(g^{-1} A g\right)$.

Proposition 4.4. If $\Gamma$ is a countable group, then $\mathbb{C}(\Gamma)$ is amenable as a $\mathbb{C}$-algebra if and only if $\Gamma$ is an amenable group. Moreover $\Gamma$ acts on $l^{2}(\Gamma)$ in an algebraically amenable fashion if and only if $\Gamma$ is an amenable group.

Proof. If $\Gamma$ is amenable, then $k(\Gamma)$ is amenable for any field $k[5]$. Now, let us suppose that $\Gamma$ is non-amenable. If $\mathbb{C}(\Gamma)$ were amenable, then the natural representation of $\Gamma$ on $\mathbb{C}(\Gamma)$ would be algebraically amenable. Therefore the natural representation of $\Gamma$ by translations on the Hilbert-space $l^{2}(\Gamma)$ must be algebraically amenable as well. By our previous proposition, there exists a bounded linear functional $\tau: B\left(l^{2}(\Gamma)\right) \rightarrow \mathbb{C}$ such that $\tau(A)=\tau\left(g^{-1} A g\right)$, for any $A \in B(\mathcal{H}), g \in \Gamma$. Consider the commutative $C^{*}$-algebra $l^{\infty}(\Gamma) \subseteq B\left(l^{2}(\Gamma)\right)$ acting on $l^{2}(\Gamma)$ by pointwise multiplication. That is, if $f \in l^{\infty}(\Gamma), G \in l^{2}(\Gamma)$,

$$
T_{f}(G)(\delta)=f(\delta) G(\delta) .
$$

The group $\Gamma$ acts on both $l^{\infty}(\Gamma)$ and $l^{2}(\Gamma)$ by translations:

$$
(\gamma f)(\delta)=f\left(\gamma^{-1} \delta\right) .
$$

Lemma 4.5.

$$
T_{\gamma f}=\gamma \circ T_{f} \circ \gamma^{-1} .
$$


Proof. Let $1_{\delta} \in l^{2}(\Gamma)$ be the function which vanishes everywhere except at $\delta$ where it takes the value 1 . Then $\gamma^{-1}\left(1_{\delta}\right)=1_{\gamma^{-1} \delta}$ and $\left(T_{f} \circ \gamma^{-1}\right)\left(1_{\delta}\right)=f\left(\gamma^{-1} \delta\right) \cdot 1_{\gamma^{-1} \delta}$. Thus $\left(\gamma \circ T_{f} \circ \gamma^{-1}\right)\left(1_{\delta}\right)=f\left(\gamma^{-1} \delta\right) 1_{\delta}$.

On the other hand, $T_{\gamma f}\left(1_{\delta}\right)=(\gamma f)(\delta) \cdot 1_{\delta}=f\left(\gamma^{-1} \delta\right) 1_{\delta}$.

Hence, $\tau$ would define a translation invariant linear functional on $l^{\infty}(\Gamma)$, which takes the value 1 on the constant one function. This is in contradiction with the non-amenability of the group $\Gamma$. Thus our proposition holds.

Remark. At the 2003 Gaeta Conference, Zelmanov mentioned the following conjecture: If a discrete group of Kazhdan's property $(T)$ is represented on an infinitedimensional vector space without a finite-dimensional invariant subspace, then the representation is not algebraically amenable.

If the action in the conjecture is in fact unitary, then by the result of [3, the representation cannot be amenable in the sense of Bekka. Hence by Proposition 4.2 . Zelmanov's conjecture holds for unitary representations.

Now, we are in the position to construct the non-amenable skew field. Let us consider the von Neumann algebra $W\left(\mathbb{F}_{2}\right)$ of the free group of two generators. The algebra $W\left(\mathbb{F}_{2}\right)$ satisfies the Ore-condition with respect to its non-zero divisors and thus it imbeds in the ring of affiliated operators $U\left(\mathbb{F}_{2}\right)$. By Lemma 10.51 of $[9$ ] there exists a skew field $D\left(\mathbb{F}_{2}\right)$ such that $\mathbb{C}\left(\mathbb{F}_{2}\right) \subseteq D\left(\mathbb{F}_{2}\right) \subseteq U\left(\mathbb{F}_{2}\right)$, namely the division closure of $\mathbb{C}\left(\mathbb{F}_{2}\right)$ in $U\left(\mathbb{F}_{2}\right)$.

Proposition 4.6. $D\left(\mathbb{F}_{2}\right)$ is a non-amenable skew field.

Proof. Recall [9] that the von Neumann algebra can be identified with the dense linear subspace of $l^{2}\left(\mathbb{F}_{2}\right)$ consisting of those vectors $w \in l^{2}\left(\mathbb{F}_{2}\right)$ such that the convolution by $w$ defines a bounded operator on $l^{2}(\Gamma)$. Thus the natural action of the group $\mathbb{F}_{2}$ on the vector space $W\left(\mathbb{F}_{2}\right)$ must be non-amenable. Since $\mathbb{C}\left(\mathbb{F}_{2}\right) \subseteq D\left(\mathbb{F}_{2}\right) \subseteq U\left(\mathbb{F}_{2}\right)$, it is enough to prove that the action of $\mathbb{F}_{2}$ on $U\left(\mathbb{F}_{2}\right)$ is not algebraically amenable. Let $V_{n} \subseteq U\left(\mathbb{F}_{2}\right)$ be a sequence of linear subspaces such that for any $g \in \mathbb{F}_{2}$,

$$
\lim _{n \rightarrow \infty} \frac{\operatorname{dim}_{\mathbb{C}}\left(g V_{n}+V_{n}\right)}{\operatorname{dim}_{\mathbb{C}}\left(V_{n}\right)}=1 .
$$

Then by the Ore-property, there exists non-zero divisors $s_{n} \in W\left(\mathbb{F}_{2}\right)$ such that $V_{n} s_{n} \subseteq W\left(\mathbb{F}_{2}\right)$. Then

$$
\lim _{n \rightarrow \infty} \frac{\operatorname{dim}_{\mathbb{C}}\left(g V_{n} s_{n}+V_{n} s_{n}\right)}{\operatorname{dim}_{\mathbb{C}}\left(V_{n} s_{n}\right)}=1 .
$$

This would mean the action of $\mathbb{F}_{2}$ on $W\left(\mathbb{F}_{2}\right)$ is algebraically amenable, leading to a contradiction.

Linnell [8] proved that $D\left(\mathbb{F}_{2}\right)$ is the free field. This finishes the proof of our Theorem 1

\section{REFERENCES}

[1] A. M. Aizenbud, On the GK-transcendence degree of division rings of fractions of polycyclic group rings, Comm. Algebra 22 (1994) no. 1, 243-251 MR.1255681 (94k:16046)

[2] M. E. B. Bekka, Amenable unitary representations of locally compact groups, Inventiones Math. 100 (1990) no. 2, 383-401. MR1047140(91g:22007)

[3] M. E. B. Bekka and A. Valette, Kazhdan's property (T) and amenable representations, Math Z. 212 (1993), no. 2, 293-299. MR1202813 (94a:22006) 
[4] P. M. Cohn, Skew fields. Theory of general division rings, Encyclopedia of Mathematics and its Applications, Cambridge University Press 57 (1995)

[5] G. Elek, The amenability of affine algebras, Journal of Algebra 264 (2003), 469-478. MR1981416 (2004d:16043)

[6] I. M. Gelfand and A. A. Kirillov, Sur les corps liés aux algèbres enveloppantes des algèbres de Lie, Inst. Hautes Études Sci. Publ. Math. 31 (1966), 5-19. MR0207918 (34:7731)

[7] M. Gromov, Endomorphisms of symbolic algebraic varieties, J. Eur. Math. Soc. 1 (1999), no. 2, 109-197. MR 1694588 (2000f:14003)

[8] P. A. Linnell, Division rings and group von Neumann algebras, Forum Math. 5 (1993), 561576. MR.1242889 (94h:20009)

[9] W. Lück, $L^{2}$-invariants: theory and applications to geometry and $K$-theory, Ergebnisse der Matematik and ihrer Grenzgebiete, Springer-Verlag, Berlin 44 (2002). MR1926649 (2003m:58033)

[10] L. Makar-Limanov, The skew field of fractions of the Weyl algebra contains a free noncommutative subalgebra, Comm. Algebra 11 (1983), no. 17, 2003-2006. MR0709019 $(84 \mathrm{j}: 16012)$

Mathematical Institute of the Hungarian Academy of Sciences, P.O. Box 127, H-1364 BudAPEST, Hungary

E-mail address: elek@renyi.hu 